\title{
Interactive and Explainable Point-of-Interest Recommendation using Look-alike Groups
}

\author{
Behrooz Omidvar-Tehrani, Sruthi Viswanathan, Jean-Michel Renders \\ firstname.lastname@naverlabs.com \\ NAVER LABS Europe \\ $h t t p: / / w w w . e u r o p e . n a v e r l a b s . c o m$
}

\begin{abstract}
Recommending Points-of-Interest (POIs) is surfacing in many locationbased applications. The literature contains personalized and socialized POI recommendation approaches which employ historical check-ins and social links to make recommendations. However these systems still lack customizability (incorporating session-based user interactions with the system) and contextuality (incorporating the situational context of the user), particularly in cold start situations, where nearly no user information is available. In this paper, we propose LikeMind, a POI recommendation system which tackles the challenges of cold start, customizability, contextuality, and explainability by exploiting look-alike groups mined in public POI datasets. LikeMind reformulates the problem of POI recommendation, as recommending explainable look-alike groups (and their POIs) which are in line with user's interests. LikeMind frames the task of POI recommendation as an exploratory process where users interact with the system by expressing their favorite POIs, and their interactions impact the way look-alike groups are selected out. Moreover, LikeMind employs "mindsets", which capture actual situation and intent of the user, and enforce the semantics of POI interestingness. In an extensive set of experiments, we show the quality of our approach in recommending relevant look-alike groups and their POIs, in terms of efficiency and effectiveness.
\end{abstract}

\section{INTRODUCTION}

There has been a meteoric rise in the use of location-based systems to benefit from services such as bike sharing [1], localized advertising [2], urban emergency management [3], and regional health-care [4]. Point-of-Interest (POI) recommendation is one of the most prominent applications of location-based services which benefit both consumers and enterprises. The task of POI recommendation is to recommend a user the POIs (e.g., restaurants, coffee shops, museums) that they may be interested in, but have never visited in a given time window. While POI recommendation in general inherits the large body of work in the community of recommender systems [5], it also carries new constraints and challenges that may not be the case for a traditional recommender, such as spatial distance semantics between POIs and user interactions on maps to select favorite POIs. An ideal POI recommendation approach should typically capture the following aspects (A1 to A4).

A1: Personalization. First, POI recommendations should be personalized, i.e., the results should be based on user preferences captured in the form of user's historical check-ins and interests.

A2: Socialization. People trust like-minded users and base their decisions on what people like them appreciated before [6]. Hence the POI recommendation should also incorporate social aspects and reflect the preferences of other people similar to the user.

A3: Customization. Beyond being personalized, the POI recommendation system should also be exploratory to incorporate user's interactions with the system, i.e., the customization of recommended POIs. This aspect calls for exploratory scenarios where the user does not have a precise POI searching criterion in mind, and wants to navigate POIs, and ultimately land on a decision after a few iterations [7].

A4: Contextualization. The POI recommendation should also capture the current situation of the user (aka context). While the literature focuses on time and location as the context, user's actual situation and intent have received less attention [8].

To the best of our knowledge, no POI recommendation approach in the literature addresses all the aforementioned aspects, A1 to A4, simultaneously. Personalization (A1) has been the focus of many approaches in the past ([9-11], to name a few), where historical check-ins are exploited to predict which POI the user prefers to visit next, using techniques such as Matrix Factorization and Poisson Factor Model. Also, socialization (A2) has been addressed in the literature $([12,13]$, to name a few $)$, where information encapsulated in location-based social networks (LBSN) are employed to predict user's preferences using link-based methods [14]. Below we discuss fundamental challenges for materializing a system which incorporates all the aforementioned aspects.

C1: Cold start. The problem of cold start arises when a user with a non-existent or limited history of check-ins asks for recommendations. A typical recommendation system which relies on historical check-ins and user similarities for personalized and socialized recommendations (A1 and A2, respectively) is unable to output results in the presence of cold start. There are three kinds of users which may cause a cold start: (i) a new user with no history, (ii) a casual user who has not visited the service for a long time, and ( $i i$ i) private users who do not want their data to be exploited. Note that the cold start problem refers to the lack of user history and not the lack of user signals, such as user's current time and location. Hence a POI recommendation system can benefit from those available signals to surmount the cold start.

C2: Interpretations of interactions. Most POI recommendation systems assume the process to be one-shot, where the user enters the system with a clear unambiguous intent, and the system returns the most interesting POIs which relates to that intent. In practice, this architecture is not realistic. Users need to interact with the system to gradually build their intent. The challenge with multishot recommendation systems (A3) is that it is not clear how user interactions with the system should influence the recommendation. 


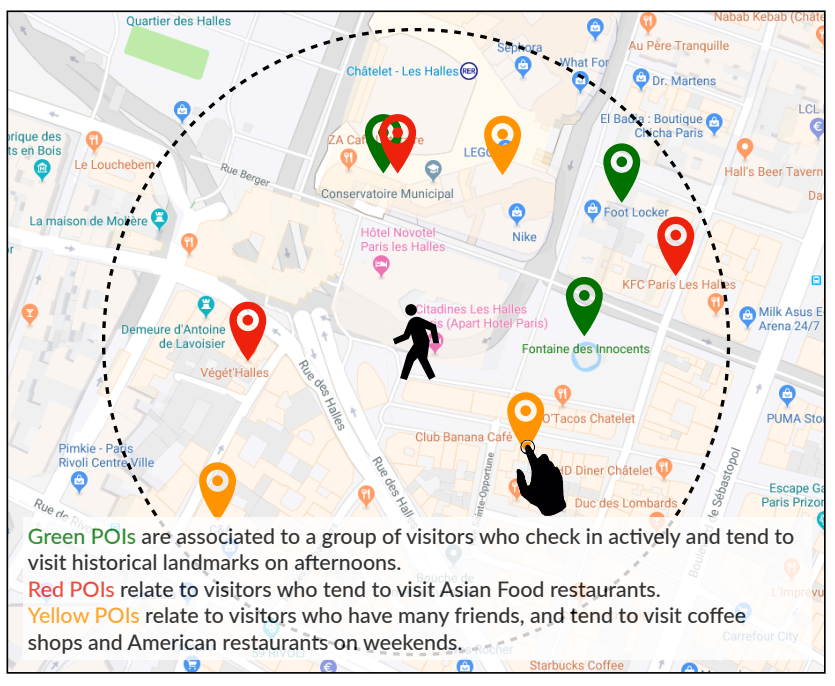

Figure 1: LikeMind in practice.

C3: Context integration. Another challenge is to integrate the context of the user into the recommendation process (A4). The context is not limited to time and location, but also to the user's mindset at the time of receiving recommendations. For instance, different POIs should be recommended in case the user is hungry, or in case he/she is seeking some personal relaxation time (i.e., me time).

C4: Explainability. Users may not trust what they get from the recommender, i.e., algorithmic anxiety due to the cold start problem (A1) and session-based interactions with the system (A3) [15]. Hence it is of crucial importance to let users know why they receive certain POIs as recommendation results.

To collectively address the challenges $\mathbf{C} \mathbf{1}$ to $\mathbf{C 4}$, we propose LikeMind, an interactive and explainable POI recommendation system based on look-alike groups. The intuition behind LikeMind is as follows: while it is assumed that no data is available from the user, POI recommendations can be obtained by finding look-alike groups in publicly available POI datasets such as YeLP ${ }^{1}$, FoursouARE ${ }^{2}$, JiePAng $^{3}$, Facebook Places ${ }^{4}$, and Gowalla ${ }^{5}$. It is shown in the literature that users trust their peers and get inspired by them for decision making [6]. The recommended POIs are explainable using their associated groups, e.g., "the group of photoholics tends to visit Montmartre in the 18th district of Paris", and "the group of food lovers tends to visit the restaurant 'les Apotres de Pigalles', in the same region." The user will then interact with those groups to detect with which group he/she identifies. As a result of this interaction, new groups will be mined, to align with the user's preferences. This iterative process ensures that groups and their POIs reflect user's interests. Note that LikeMind discovers user's interests and aligns recommendations accordingly, without the need of any historical

\footnotetext{
$\overline{{ }^{1} \text { https://www.yelp.com/dataset }}$

${ }^{2}$ https://developer.foursquare.com/docs/places-database/

${ }^{3}$ https://jiepang.com

${ }^{4} \mathrm{https}: / / w w w$ facebook.com/places

${ }^{5}$ https://snap.stanford.edu/data/loc-gowalla.html
}

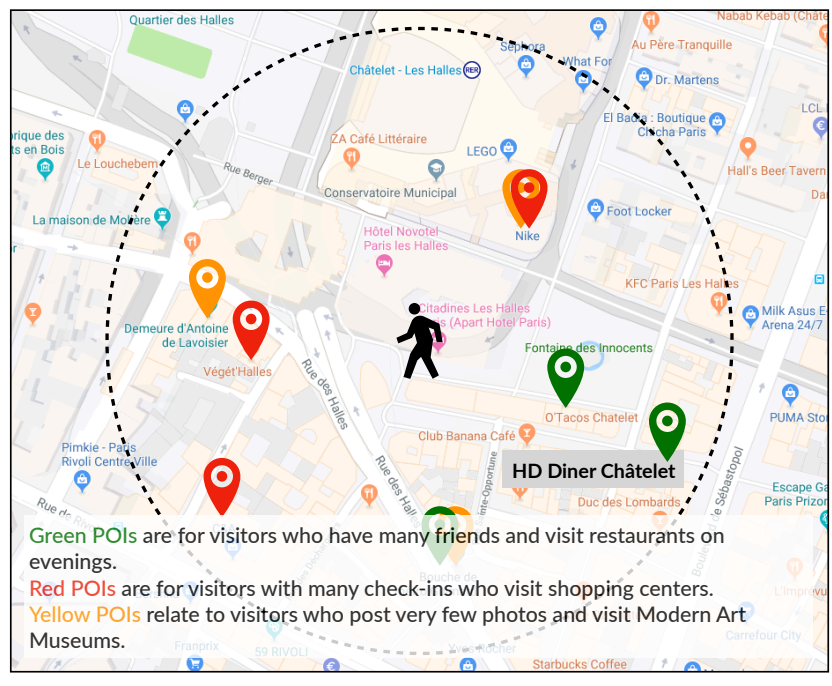

Figure 2: Followup iteration in LikeMind after clicking a POI from the yellow group in Figure 1.

check-in data from the user. The following example describes how LikeMind functions in practice.

EXAMPLE 1. Jane is visiting Paris as a tourist. She is walking in the area of the Pompidou center. After 30 minutes of walking, she gets tired and asks LikeMind for "me time" recommendations, to find POIs in her vicinity (the dashed circle in Figure 1) where she can sit and relax. Fane is concerned about privacy and does not share any historical check-in data with the system. LikeMind outputs three user groups related to fane's intent, and top-three POIs for each group (Figure 1). She looks at group descriptions to see where she can find some doppelgängers in group members. Being a social person, fane shows interest in the yellow group, i.e., visitors who have many friends (i.e., social visitors like her), and tend to visit coffee shops and American restaurants on weekends. This motivates her to reformulate her intent and ask for a place where she can eat. She interacts with the system and asks where people usually eat in the neighborhood. Hence LikeMind returns another three groups to satisfy Jane's intent (Figure 2). This helps fane to make up her mind and go to an American-cuisine restaurant.

The example shows that interactive and explainable recommendations help users refine their ambiguous needs and finalize their decision making process on POI selection. The way users can specify their mindsets (e.g., "me time", "I'm hungry") enables users enforce their context to the recommendation system (i.e., tackling C3) and steer the results towards what they are really interested to receive.

Contributions. In summary, we propose the following contributions.

- We address the cold start and explainability challenges by employing look-alike user data as a proxy for user preferences. We reformulate the problem of POI recommendation, as recommending explainable groups (and their POIs) which are in line with user's interests. 
- We consider "recommendation" as an exploratory process where the user interacts with the system, and his/her interactions impact the way look-alike groups are selected out. We mention how we build a user portfolio of POIs in order to make recommendations more relevant in further iterations.

- We introduce and formalize the notion of "mindsets", which captures actual situation and intents of the user. We mention how the interestingness of POIs is maximized using mindsets.

- In an extensive set of quantitative and qualitative experiments, we show the efficiency and effectiveness of LikeMind.

The outline of the paper is as follows: in Section 2, we provide the data model. In Section 3, we formally define our problem and discuss the challenges. In Section 4, we describe LikeMind architecture and its underlying algorithm as a solution for interactive and explainable POI recommendation. Section 5 presents the detailed experiments. The related work is provided in Section 6. Last, we conclude and discuss future directions in Section 7.

\section{DATA MODEL}

We consider a user $\mu$ asking for POI recommendations. We denote $\mu$ 's portfolio as a set $\mathcal{P}_{\mu}$ of POIs that $\mu$ is interested in. Following our assumption of cold start (C1), we consider that initially $\mathcal{P}_{\mu}=\emptyset$. Additionally, we consider a POI dataset $\mathcal{D}=\langle\mathcal{U}, \mathcal{P}\rangle$ with a set of visitors $\mathcal{U}$ and a set of POIs $\mathcal{P}$.

Visitors. For a visitor $u \in \mathcal{U}$, the set $u$.demogs contains tuples of the form $\langle d, v\rangle$ where $d$ is a demographic attribute (e.g., age, gender, number of trips, number of check-ins), and $v \in \operatorname{domain}(d)$. Also the set $u$.checkins contains tuples of the form $\langle p, t\rangle$ which represents that $u$ has visited a POI $p \in \mathcal{P}$ at time $t$.

POIs. A POI $p \in \mathcal{P}$ is defined as a tuple $p=\langle$ loc, att $\rangle$ where $p$.loc is itself a tuple $\langle$ lat, lon $\rangle$ (latitude and longitude, respectively) which defines where $p$ is situated geographically. The set $p$.att is a set of tuples of the form $\langle a, v\rangle$ which denote that the POI has the value $v$ for the attribute $a$, such that $v \in \operatorname{domain}(a)$.

We measure the interestingness of POIs using POI utility functions. A more interesting POI has higher chances to be recommended to the user $\mu$. A POI utility function $f: 2^{\mathcal{P}} \mapsto[0,1]$ returns a value between 0 and 1 which reflects the extent of interestingness for one or several POIs [16]. Table 1 lists POI utility functions that we employ in this work. We denote the set of all POI utility functions as $\mathcal{F}$. We also define all utility functions as maximization objectives.

\section{PROBLEM DEFINITION}

We form our problem definition based on two core assumptions. First, we assume that the user $\mu$ is in an exploratory setting and does not necessarily have a clear idea of his/her needs [7], and the user is going to sharpen his/her intent in several iterations [17]. Second, we conjecture that look-alike user data is a good proxy to gain user preferences [18], in the absence of historical check-ins of $\mu$. These two assumptions enable us to define the problem according to the challenges $\mathbf{C} \mathbf{1}$ to $\mathbf{C} \mathbf{4}$ presented in Section 1. In this section, first we discuss how the aforementioned assumptions are incorporated in the problem of interactive and explainable POI recommendation. Then we formally define our problem and discuss its hardness.

\begin{tabular}{|l|l|}
\hline Utility function & Description \\
\hline \hline popularity $(P)$ & $\begin{array}{l}\text { normalized average number of } \\
\text { POI check-ins in } P \text {. }\end{array}$ \\
\hline prestige $(P)$ & $\begin{array}{l}\text { normalized average rating } \\
\text { score of POIs in P. }\end{array}$ \\
\hline recency $(P)$ & $\begin{array}{l}\text { inverse difference between the } \\
\text { current date and the average } \\
\text { insertion date of POIs in } P .\end{array}$ \\
\hline coverage $(P)$ & $\begin{array}{l}\text { the area of the convex hull induced by } \\
\text { the geographical location of POIs in } P \\
\text { normalized by the size of the city. }\end{array}$ \\
\hline surprisingness $(P)$ & $\begin{array}{l}\text { normalized Jaccard distance between } \\
\text { POI categories of } P \text { and POI } \\
\text { categories of the visited POIs } \\
\text { by the user in } \mathcal{P} \mu \text {. }\end{array}$ \\
\hline category $(P$, cat $)$ & $\begin{array}{l}\text { normalized Jaccard similarity } \\
\text { between the set cat and the } \\
\text { POI categories in } P .\end{array}$ \\
\hline diversity $(P)$ & $\begin{array}{l}\text { normalized Jaccard distance } \\
\text { between sets of POI categories in } P .\end{array}$ \\
\hline size $(P)$ & $\begin{array}{l}\text { normalized average radius } \\
\text { of POIs in } P \text {. }\end{array}$ \\
\hline
\end{tabular}

Table 1: POI utility functions $(P \subseteq \mathcal{P})$.

\subsection{Exploratory settings}

Context. A core concept in an exploratory POI recommendation setting is "context", which is often materialized as the current time and location of the user, denoted as a tuple $c_{\mu}=\langle$ loc, time $\rangle$ for a given user $\mu$. For more generality, we often represent $c_{\mu}$.time as a categorized variable. ${ }^{6}$ An additional dimension of contextuality is "mindset", i.e., the actual situation and intent of the user.

Mindsets. Mindsets should reflect the way interestingness of POIs are computed based on user's interests. While online services such as AroundME ${ }^{7}$ enable users to explore their nearby region by selecting explicit POI categories (e.g., museums, historical landmarks), mindsets capture implicit intents of users (e.g., "let's learn") which are more challenging to capture. A mindset $m$ is a tuple $m=\langle$ label, $f u n c()\rangle$, where label provides a short description of the mindset, and $f u n c()$ defines semantics of POI interestingness. For instance, in case $m$.label = "let's learn", m.func() favors museums, libraries and cultural landmarks. We denote the set of all mindsets as $\mathcal{M}$.

Materializing mindsets. In a field study, with a prototype of a POI recommendation system, we performed an initial concept validation using the Wizard of $\mathrm{Oz}$ methodology to discover how users perceive exploring POIs using different mindsets (see [19] for the details of our field study). We recruited a diverse set of 12 participants for an in-depth analysis of their situation and intent for receiving POI recommendations. During these sessions with our participants, we presented them with 7 different mindsets, which we had initially hypothesized. Our participants were first asked to choose a mindset, which would in turn lead them to find a POI

\footnotetext{
${ }^{6}$ We consider the following categories for time: "morning" (5AM-11AM), "afternoon" (12PM-5PM), "evening" (6PM-10PM), and "night" (11PM-4AM).

${ }^{7}$ http://www.aroundmeapp.com
} 


\begin{tabular}{|l|l|}
\hline Mindset label & Description \\
\hline$m_{1}:$ I'm new here & $\begin{array}{l}\text { towards touristic POIs about the } \\
\text { popular attractions in the city. }\end{array}$ \\
\hline$m_{2}:$ surprise me & $\begin{array}{l}\text { towards POIs which haven't been } \\
\text { visited before by the user and are } \\
\text { uncommon (seldom visited) }\end{array}$ \\
\hline$m_{3}:$ let's workout & $\begin{array}{l}\text { towards POIs related to physical } \\
\text { exercises like swimming pools, } \\
\text { parks, gyms, and mountains }\end{array}$ \\
\hline$m_{4}:$ me time & $\begin{array}{l}\text { towards POIs related to activities } \\
\text { to treat oneself and be pursued solo } \\
\text { to unwind and relax }\end{array}$ \\
\hline$m_{5}:$ I'm hungry & $\begin{array}{l}\text { towards getting faster access } \\
\text { to food-related POIs nearby }\end{array}$ \\
\hline$m_{6}:$ let's learn & $\begin{array}{l}\text { towards POIs such as museums, } \\
\text { libraries and cultural landmarks }\end{array}$ \\
\hline$m_{7}:$ hidden gems & $\begin{array}{l}\text { towards intriguing local POIs } \\
\text { that are highly rated but } \\
\text { not necessarily popular }\end{array}$ \\
\hline
\end{tabular}

Table 2: Mindsets

they would like to visit. In this study, we were able to qualitatively confirm the usefulness of mindsets to find POIs. We meticulously paraphrased our participants' interpretation of the mindsets to reach descriptions provided in Table 2 . While these mindsets are the result of an in-depth study of POI exploration needs, our model is generic enough to allow new mindsets. We describe the process of mindset creation in Section 4.4.

Mindset function. Given a mindset $m$, the function $m$.func() is defined as follows.

$$
m . f u n c(P, \mu)=\frac{\sum_{f_{i} \in \mathcal{F}} w_{i, \mu} b_{i, m} f_{i}(P)}{\sum_{f_{i} \in \mathcal{F}} w_{i, \mu} b_{i, m}}
$$

In Equation $1, f_{i}(P)$ is a utility function (see Table 1 ) applied on a set of POIs $P \subset \mathcal{P}$, and $w_{i, \mu}$ and $b_{i, m}$ are the user-specific weight and the prior of the function $f_{i}$ for the user $\mu$ and the mindset $m$, respectively. Priors reflect the importance of a utility function for a mindset. In case $b_{i, m}=0$, it means that the function $f_{i}$ has no influence on the mindset $m$. On the contrary, in case $b_{i, m}=1$, it means that the mindset $m$ is defined only based on $f_{i}$. Userspecific weights, on the other hand, reflect the importance of a utility function for the user. A user may have, for instance, more interest in popularity than coverage. The weights are assumed to shape up when the user interacts with the system. Given the set of all possible user-specific weights $W$, we initially set $\forall w \in W, w=$ 1.0. While weights are dynamic and changes per user, priors can be learned offline and stay unchanged at the online execution.

Utility function priors. Table 3 shows a consensus over our field study [19] on utility function priors in mindsets. For instance, the mindset $m_{2}$ (i.e., "surprise me") is a combination of the following utility functions in decreasing order of their prior value: surprisingness, popularity, prestige, diversity, and size. In case of the category function, we need to specify POI categories of interest. Table 4 shows these specific categories for each mindset. Note that $m_{1}$, $m_{2}$, and $m_{7}$ are not related to the category function, hence are excluded from Table 4 . While these categories come from the Gowalla dataset, similar set of categories exists in other POI datasets as well.

\subsection{Look-alike user data}

There exist various publicly available POI datasets structured as $\mathcal{D}=\langle\mathcal{U}, \mathcal{P}\rangle$. To build look-alike relations in a publicly available POI dataset, we build "visitor groups" which aggregate a set of visitors with common demographics and/or POIs [18]. Grouping visitors is in conformance with the "human mobility behavioral clustering phenomenon" which mentions that individual visiting locations tend to cluster together [20]. Visitor groups are obviously virtual and group members do not necessarily know each other. In other words, members of a group are "location friends" (who have checked in the same places) and not "social friends" (who are socially connected in an LBSN) [13]. A visitor group is a triple $g=$ $\langle$ members, demogs, POIs $\rangle$ where g.members $\subseteq \mathcal{U}$, and "demogs" and "POIs" contain following expression-based conditions that those members should satisfy:

- (i.) $\forall u \in$ g.members, $\forall\langle a, v\rangle \in$ g.demogs, $\langle a, v\rangle \in$ u.demogs,

- (ii.) $\forall u \in$ g.members, $\forall p \in$ g.POIs, $\exists\langle p, t\rangle \in$ u.checkins.

We also define the relevance between a visitor group $g$ and $\mathcal{P}_{\mu}$ as a function $\operatorname{rel}\left(g, \mathcal{P}_{\mu}\right)$ defined below.

$$
\operatorname{rel}\left(g, \mathcal{P}_{\mu}\right)= \begin{cases}\frac{\left|\mathcal{P}_{\mu} \cap g . P O I s\right|}{\left|\mathcal{P}_{\mu}\right|} & \mathcal{P}_{\mu} \neq \emptyset \\ 1 & \text { otherwise }\end{cases}
$$

Intuitively, a group $g$ is relevant to a user $u$ iff there exist some common POIs between the user $u$ and the group $g$. In case $\mathcal{P}_{\mu}=\emptyset$ (i.e., cold start), we assume that $g$ is relevant whatsoever.

\subsection{Formal problem definition}

We define our problem as follows. Given a user $\mu$ and his/her affiliated context $c_{\mu}=\langle$ loc, time $\rangle$, a mindset $m=\langle$ label, func $\rangle$, a radius $r$, a relevance threshold $\sigma$, and integers $k$ and $k^{\prime}$, the problem is to find $k$ groups $G$ and $k^{\prime}$ POIs for each group in $G$, such that the following conditions are met.

- (i.) $\forall g \in G, g, \operatorname{rel}\left(g, \mathcal{P}_{\mu}\right) \geq \sigma$;

- (ii.) $\forall g \in G, \forall p \in$ g.POIs, distance $\left(p . l o c, c_{\mu} . l o c\right) \leq r$;

- (iii.) $\forall g \in G, \forall p \in g . P O I s, \forall u \in g$.members,

$\forall\langle p, t\rangle \in$ u.checkins, $t=c_{\mu}$.time;

- (iv.) $\Sigma_{g \in G}($ m.func $(g . P O I s, \mu))$ is maximized.

The first three conditions ensure that groups are relevant to the user, in vicinity of the user's location, and in the same time category of the user's context. The last condition applies the input mindset to groups, and verifies whether groups' POIs are maximally in line with the mindset.

Problem hardness. Appendix A shows the theoretical hardness of the aforementioned problem. The problem is also challenging in practice, because the potential number of relevant groups is huge and hence the mindset maximization is not straightforward. Given the complexity of formulating look-alike groups and mindsets in the task of POI recommendation, we consider set-based semantics for our recommendation problem. We plan to consider more complex 


\begin{tabular}{|l|c|c|c|c|c|c|c|c|}
\hline & popularity & prestige & recency & coverage & surprisingness & category & diversity & size \\
\hline \hline$m_{1}:$ I'm new here & $\mathbf{0 . 2 5}$ & $\mathbf{0 . 2 5}$ & 0.10 & 0.15 & 0.00 & 0.00 & $\mathbf{0 . 2 5}$ & 0.00 \\
\hline$m_{2}:$ surprise me & 0.25 & 0.20 & 0.00 & 0.00 & $\mathbf{0 . 3 0}$ & 0.00 & 0.15 & 0.10 \\
\hline$m_{3}$ : let's workout & 0.25 & 0.25 & 0.00 & 0.10 & 0.00 & $\mathbf{0 . 4 0}$ & 0.00 & 0.00 \\
\hline$m_{4}:$ me time & 0.10 & 0.10 & 0.00 & 0.10 & 0.00 & $\mathbf{0 . 4 0}$ & 0.00 & 0.30 \\
\hline$m_{5}:$ I'm hungry & 0.05 & 0.20 & 0.10 & 0.15 & 0.00 & $\mathbf{0 . 4 0}$ & 0.05 & 0.05 \\
\hline$m_{6}$ : let's learn & 0.20 & 0.20 & 0.00 & 0.10 & 0.00 & $\mathbf{0 . 4 0}$ & 0.10 & 0.00 \\
\hline$m_{7}$ : hidden gems & $\mathbf{0 . 3 0}$ & $\mathbf{0 . 3 0}$ & 0.15 & 0.00 & 0.00 & 0.00 & 0.00 & 0.25 \\
\hline
\end{tabular}

Table 3: Priors in mindsets. The largest values of prior per mindset are shown in bold.

\begin{tabular}{|l|l|}
\hline Mindset & Categories of interest \\
\hline$m_{3}$ : let's workout & sport fields, park, health and fitness, bowling, tennis court, ice skating, gym \\
\hline$m_{4}:$ me time & outdoor, food, tea room, bar, coffee shop \\
\hline$m_{5}$ : I'm hungry & food, restaurant \\
\hline$m_{6}$ : let's learn & museum, art, gallery, library sculpture, bookstore, movie theater, historical landmark, monument \\
\hline
\end{tabular}

Table 4: Categories of interest for mindsets

problems in our immediate future work, e.g., POI sequence recommendation [21,22] and travel package recommendation $[23,24]$.

\section{ALGORITHM}

LikeMind is a session-based system which begins with an ambiguous user's intent for POI recommendation, and ends when he/she is satisfied with the resulting POIs. Each session consists of a finite sequence of iterations which captures interactions with the user. A new iteration begins by defining a mindset (which may remain the same as the previous iteration), which then results in $k$ relevant groups and $k^{\prime}$ POIs for each group. At the end of each iteration, the user is free to bookmark some of the recommended POIs as his/her favorites. Hence there are two types of feedback that the user can provide to the system: the mindset and POI bookmarks. This multi-shot architecture (i.e., A3) contradicts most traditional single-shot POI recommendation approaches by incorporating user interactions in the recommendation (i.e., addressing $\mathbf{C} 2$ ). Note that the input mindset at each iteration is part of the user signal and not user history, and LikeMind exploits mindsets to surmount the cold start. $^{8}$

LikeMind iterations. At each iteration, the system returns groups and POIs from a POI dataset (addressing the cold start challenge C1) based on the functionality of the selected mindset. Algorithm 1 describes the process in each single iteration:

Step 1: Neighborhood filtering. First, the system finds all the nearby POIs which are at most $r$ kilometers/miles far from the user (line 1), where $r$ is a user-defined input parameter. The parameter $r$ enforces the Tobler's First Law of Geography [20] and ensures that recommendations relate to user's location (i.e., C3). We employ an efficient implementation of ST_DWithin function in PostGIS to achieve a near real-time retrieval of POIs. We denote the set of nearby POIs as $P \subseteq \mathcal{P}$.

Step 2: Check-in retrieval. Given $P$, LikeMind then retrieves all checkins of the nearby POIs (line 2). We denote the set of all nearby checkins as $H$. A check-in $\langle p, t\rangle$ is in $H$ iff $p \in P$ and $t=c_{\mu}$.time. The second condition conveys that the check-in and the user context

${ }^{8}$ One direction of our future work is to automatically predict mindsets based on other available user signals. should belong to the same time category, e.g., "morning". LikeMind finds which visitors checked in nearby POIs using check-ins.

Step 3: Group mining. Then the system mines groups among checkedin visitors (line 3) denoted as $G^{*}$. Given that $\left|G^{*}\right| \gg k$, the system finds $k$ groups $G \subset G^{*}$ (s.t., $|G|=k$ ) which collectively maximize the mindset function (line 4). The group set $G$ is henceforth aligned to the user's intent expressed in the mindset.

Step 4: POI selection. Finally, LikeMind picks top- $k^{\prime}$ POIs for each group which are visited by most of the group members (line 5). At this stage, the user observes $k$ groups and $k^{\prime}$ POIs for each, and may decide to bookmark POIs for enriching her portfolio.

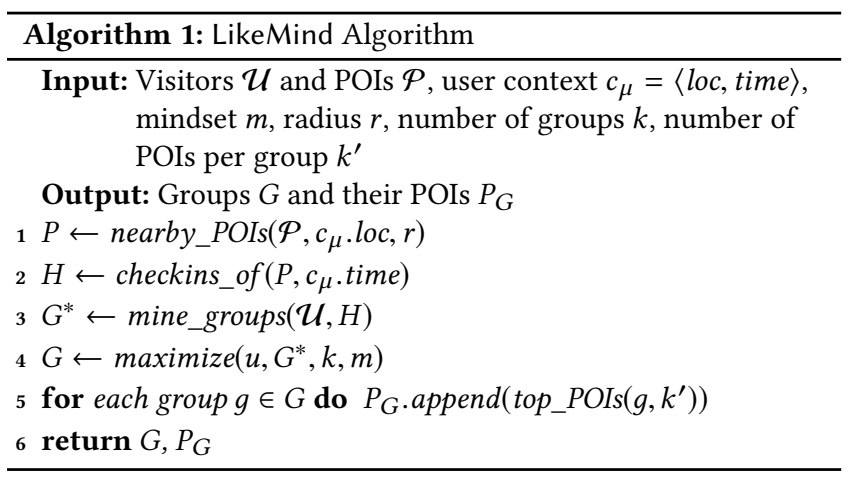

In the following, we discuss how groups are mined (i.e., the mine_groups() function in line 3), how mindset functions are maximized (i.e., the maximize() function in line 4), and how bookmarking POIs improves the recommendations in further iterations.

\subsection{Mining groups}

To address the challenge of explainability (C4), we aim to find describable groups which identify a set of visitors checking in a set of POIs. For this aim, we employ Frequent Itemset Mining (FIM) technique, where each group is a frequent itemset, and items are common demographic attributes and POIs of the group members. While groups can be discovered in myriad ways [18, 25, 26], we choose FIM to obtain describable groups with overlaps, so that 
visitors can be a member of more than one group and be described in different ways. For each visitor $u \in \mathcal{U}$, we build a transaction embedding trans $(u)$ which contains all demographic attributes and visited POIs of $u$. Appendix B describes the process of building and enriching the embeddings. The set $\tau$ contains the embeddings of all visitors, i.e., $\tau=\{\operatorname{trans}(u) \mid u \in \mathcal{U}\}$. Given an arbitrarily group of visitors $g=\langle$ members, demogs, POIs $\rangle$, we define the group support $\operatorname{supp}(g)$ as a measure of $g$ 's significance (Equation 3 ).

$$
\begin{aligned}
\operatorname{supp}(g)=\mid\{u \in \mathcal{U} \mid & \operatorname{trans}(u) \in \tau, \\
\text { g.demogs } & \cup g . P O I s \subseteq \operatorname{trans}(u)\} \mid
\end{aligned}
$$

The group $g$ is a frequent itemset (and hence a valid group) iff $\operatorname{supp}(g)$ is larger than a predefined support threshold. While the support threshold is often a user-defined parameter, we materialize all groups with support larger than 1 , hence no threshold needs to be specified.

Given the set $\tau$, we employ the ExTRACT algorithm [27] to mine groups. The input of the algorithm is of type ARFF (AttributeRelation File Format) which contains the set of all enriched embeddings. The frequent itemset mining algorithm is known to be inefficient for large number of transactions, as its execution time grows exponentially with the number of transactions. This is why that the algorithm is often considered as an offline step preceding an online investigation on groups. In LikeMind, we are able to perform the mining process on-the-fly, thanks to the neighborhood filters preceding the algorithm. The algorithm mines groups only for visitors with check-ins in the vicinity of the user. Hence the size of the visitor set is drastically reduced compared to $|\mathcal{U}|$.

\subsection{Maximizing mindsets}

Not all groups are equally interesting to the user. We need to pick $k$ groups out of all mined groups which are in line with the mindset requested by the user. This will tackle the challenge of context integration (C3). Each mindset is associated to a function which is a set of utility functions combined in a linear fashion with userspecific weights and priors (Equation 1). The mindset function admits as input a set of POIs, and returns a value in the range $[0,1]$. Given a mindset $m$ and a group $g$, we measure the utility of $g$ regarding $m$ 's functionality, as follows.

$$
\text { group_utility }(g)=m . f u n c(g . P O I s)
$$

Given the space of all group utility values, the problem is to find $k$ groups with the largest values of group utility. As each mindset function is constructed as a combination of several utility functions, maximizing mindset functions is a multi-objective optimization problem in nature. However, we employ a scalarization approach in LikeMind using user-specific weights and priors to reduce the complexity of the problem to single-objective optimization. One direction of future work is to directly employ multi-objective optimization approaches to obtain skyline groups.

In LikeMind, we employ a greedy-style hill climbing algorithm to maximize mindset functions (Algorithm 2):

Step 1: Pruning. The algorithm starts by removing all irrelevant groups to the user $\mu$ (line 2). This ensures that groups are inline with user's preferences provided in previous interactions. While

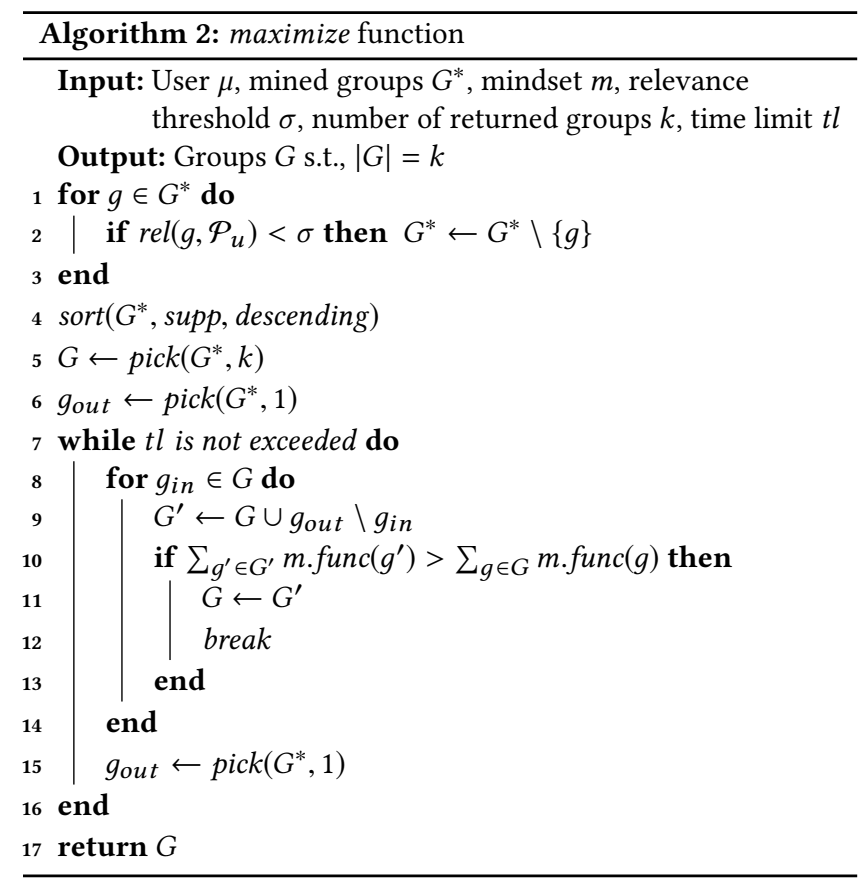

LikeMind focuses on the cold start problem and employs no historical data for building recommendations, it can however benefit from user's interactions with the system to align recommendations with his/her preferences. We enable users to describe their preferences by "bookmarking" recommended POIs. These bookmarked POIs will enrich the user's portfolio, which later contributes to identifying irrelevant groups to user's preferences. Once the user $\mu$ clicks on a POI $p$, it will be added to $\mathcal{P}_{\mu}$. Then the relevance of a group $g$ regarding $\mathcal{P}_{\mu}$, i.e., $\operatorname{rel}\left(g, \mathcal{P}_{\mu}\right)$ is computed as in Equation 2.

Step 2: Mindset function maximization. After pruning irrelevant groups, the algorithm iterates over the space of groups to maximize the mindset function. At each step, the algorithm introduces a new group to the set of $k$ groups, and verifies if the value of the mindset function increases. In case of improvement, the new group will become a member of the $k$ groups, and another group will be selected for a substitution. The improvement loop breaks when a time limit $t l$ exceeds. While the time limit $t l$ should normally be a user-defined input parameter, we inspire from "progressive analytics" [28] and fix the time to "continuity preserving latency", i.e., $t l=100 \mathrm{~ms}$, which is the the limit for humans to have an instantaneousness experience of the interactive process.

The function pick() (lines 5 and 6) may employ different semantics to enforce the "scanning order" in the space of groups $G^{*}$. The semantics is usually designed in a way to boost the optimization process by moving faster towards the optimized value [29]. In our case, mindsets are combinations of different optimization objectives. We employ the "support" measure (Equation 3) to enforce order, hence larger groups have higher chances to be selected. Larger groups contain more visitors and provide richer insights [18]. Hence the function pick $\left(G^{*}, k\right)$ returns top- $k$ unseen groups in $G^{*}$ with the largest support. 


\subsection{Improving recommendations}

The recommendations should become more personalized (addressing C1) in further interactions of the user with the system (addressing C2). To achieve this, we update weights in mindset functions according to user's interactions. Given a user $\mu$ and a mindset $m$, the weight of a function $f_{i}$ in $m$.func() is calculated as follows.

$$
w_{i, \mu}= \begin{cases}f_{i}\left(\mathcal{P}_{\mu}\right) & \mathcal{P}_{\mu} \neq \emptyset \\ 1 & \text { otherwise }\end{cases}
$$

In case $\mu$ has already performed some interactions with the system (i.e., $\mathcal{P}_{\mu} \neq \emptyset$ ), the value of $w_{i, \mu}$ is set relative to the orientation of $\mu$ 's previous choices towards $f_{i}$. It reflects the importance of a utility measure for the user $\mu$. In case no interaction is available, the weight is set to 1.0. For instance, in case $f_{i}=$ coverage, and $\mathcal{P}_{\mu}$ contains POIs only from one single neighborhood of the city, $w_{i, \mu}$ will probably receive a value close to zero. Intuitively, it shows that there is less importance in $\mu$ 's subjective preference for coverage.

It is important to notice that $\mathcal{P}_{\mu}$ impacts LikeMind in two different levels of granularity, group and mindset levels. In the group level, it enables the system to have an early pruning on groups with no POI overlap with $\mathcal{P}_{\mu}$ (line 2 of Algorithm 2). In the mindset level, it aligns user-specific weights of utility functions to user preferences in $\mathcal{P}_{\mu}$.

\subsection{Beyond predefined mindsets}

While we propose a set of mindsets in Table 2, the functionality of LikeMind is not dependent on a predefined set of mindsets and can be easily extended to new ones. A new mindset $m^{*}$ is identified by its priors $b_{i, m^{*}}$. The new mindset can be created either from the ground up, or by combining some predefined mindsets. In the former case, $m^{*}$ is derived from a set of interesting POIs $P$ provided by the user, where $b_{i, m^{*}}=f_{i}(P)$. In the latter case, $m^{*}$ is constructed as an aggregation of a subset of predefined mindsets $M \subseteq \mathcal{M}$, where $b_{i, m^{*}}=\operatorname{avg}_{m \in M}\left(b_{i, m}\right)$.

\section{EXPERIMENTS}

In this work, we use GowALLA dataset [30], collected from a popular LBSN with 36, 001, 959 check-ins of 319, 063 visitors over 2, 844, 076 POIs. GowAllA's check-in matrix density is $2.9 \times 10^{-5}$. POIs are grouped in 7 different categories, i.e., community, entertainment, food, nightlife, outdoors, shopping, and travel. GowALla is among the few POI datasets that provide attributes both for visitors and POIs. Hence we can form groups containing both demographic attributes and POIs. This increases the explainability of groups, and enables users find out in which group they identify. Visitor attributes are illustrated in Table 6. Also POIs are described using the following attributes: insertion date, location, total number of check-ins, radius, and categories. In this section, we present a comprehensive set of experiments to validate the efficiency and effectiveness of LikeMind.

Experimental methods. Unlike one-shot recommendation algorithms, an exploratory system such as LikeMind incorporates endusers in the loop, hence the need for an extensive validation of both algorithmic and user-centric aspects. Section 5.1 evaluates the overall algorithmic behavior of LikeMind by simulating interaction sessions and reporting the Hit Ratio measure. Section 5.2 evaluates the human-oriented aspects of LikeMind using an extensive user study in Amazon Mechanical Turk. A complementary viewpoint on the performance aspects of LikeMind is also provided in Appendix C.

Baselines. Note that the functionality of LikeMind is fundamentally different from the most POI recommendation approaches in the literature. First, most methods ranging from Collaborative Filtering to deep learning approaches require the checkins matrix as input, which is assumed to be nonexistent in the case of LikeMind. Second, they return a ranked list, while set-based semantics is employed in LikeMind. For a fairer comparison, we employ the two following preliminary baselines: (i) Diversity which returns a diversified set of POIs in user's vicinity, and (ii) Popularity which returns the most popular POIs.

\subsection{Simulation Study}

Our goal in this part of our experiment is to examine the overall behavior of LikeMind in tackling the cold-start problem, and providing customizability, contextuality, and explainability in POI recommendation. Preferences of real people will be verified later in the user study (Section 5.2). To remove the influence of human decisions from the exploratory process of LikeMind, we simulate interactions in the Gowalla dataset. We then report the Hit Ratio (HR) for each simulated session.

We simulate 100 different sessions and report HR as the average over all the sessions. In each session, first we randomly pick a user $\mu$ from Gowalla. From the set $\mu$.checkins, we randomly pick a check-in $\langle p, t\rangle$, and set $\mu$ 's context as $c_{\mu}=\langle p$.loc, $t\rangle$. We build a set $\zeta_{\mu} \subseteq \mu$.checkins as follows.

$$
\begin{gathered}
\zeta_{\mu}=\left\{\langle p, t\rangle \in \mu . \text { checkins } \mid \text { distance }\left(p . l o c, c_{\mu} . l o c\right) \in(0, r]\right. \\
\left.\wedge \mid t-c_{\mu} . \text { time } \mid \leq 48\right\}
\end{gathered}
$$

The set $\zeta_{\mu}$ contains nearby POIs (constrained using the radius $r$ ) that $\mu$ visited in the next two days following his/her current context time $c_{\mu}$.time. In this experiment, we fix $r=0.5 \mathrm{~km}$. LikeMind contributes to HR if its output overlaps with $\zeta_{\mu}$. To simulate the cold-start, the whole set $\mu$.checkins is masked off as testing set.

Each session contains $N$ consecutive iterations. Each iteration is fired by simulating the action of picking a mindset $m$. LikeMind will then generate groups and their POIs using $m$ and $c_{\mu}$. The iteration ends by simulating the action of picking a group of interest $g^{*}$ and a POI of interest $p^{*}$ associated with $g^{*}$. The POI $p^{*}$ will be added to $\mathcal{P}_{\mu}$. We propose two baselines for group selection: (i) pick a group at random; ( $i i)$ pick a group $g^{*}$ where Cosine $\left(g^{*}\right.$.demogs, $\mu$.demogs) is maximal. We call the latter the optimal group strategy. Moreover, we propose two baselines for mindset selection: $(i)$ pick one of the mindsets in Table 2 at random; (ii) pick the mindset $m$ with maximal value of $m$.func() for the POIs of $g^{*}$ in the previous iteration. We call the latter the optimal mindset strategy. For a more realistic simulation of the mindset selection process, we consider the fact that users do not always switch to a new mindset in each iteration. Hence we also consider a parameter $\theta$ which is the probability that the mindset in the next iteration will stay unchanged. For instance, in case $\theta=0.8$, it is highly probable that the mindset does 
not change between consecutive iterations. By default, we employ random strategy for both group and mindset selection, and $\theta=0.5$.

We report HR at two different levels of granularity, iteration level and session level. At the iteration level, we denote the measure as $H R_{I} @ N$, and compute it as the average HR obtained at each iteration in a session.

$$
H R_{I} @ N=\frac{\sum_{i=1}^{S}\left(\frac{\sum_{j=1}^{N} \mathbb{1}(i, j, \mu)}{N}\right)}{S}
$$

In Equation 7, $N$ is the number of iterations, $S$ is the number of sessions (in our case, $S=100$ ), and $\mathbb{1}(i, j, \mu)$ is an iteration-based hit indicator function which returns " 1 " if there is a hit (POI in common with $\zeta_{\mu}$ ) at iteration $j$ of the session $i$. At the session level, we denote the measure as $H R_{S} @ N$, and compute it as the average HR for the whole session, as the result of all interactions in $N$ iterations.

$$
H R_{S} @ N=\frac{\sum_{i=1}^{S}\left(\mathbb{1}_{j=1}^{N}(i, j, \mu)\right)}{S}
$$

In Equation 8, $\mathbb{1}(i, j, \mu)$ is a session-based hit indicator function which returns " 1 " if there is at least one hit in the iterations $j$ of the whole session $i$. Obviously session level HR subsumes the one at the iteration level.

Figure ?? illustrates HR values using different strategies of group selection, mindset selection, and mindset change. We measure HR by varying $N$ from 2 to 50 . The figures on the left report iteration level HR, and the ones on the right report session level HR. To reduce clutter, we don't show the baseline results on the figures, where Popularity has a constant value of 0.005 for $H R_{S} @ N$, and Diversity grows from zero to 0.001 .

Group selection strategy. We observe that optimal groups increase HR by $22 \%$ and $38.8 \%$ on average, in iteration and session levels, respectively (top row in Figure ??). In session level, the HR grows to values larger than $50 \%$ after only 10 iterations, and it reaches to $82 \%$ at 50 iterations. This confirms our assumption that look-alike groups function as a good proxy to gain user preferences.

Mindset selection strategy. We observe that optimal mindsets increase HR by $22.4 \%$ in the session level (middle row in Figure ??) However the increase in the iteration level is not salient. This shows that in contrary to group selection strategies, mindset selection has a long-term influence on the overall session. In the session level, the HR grows to values close to $50 \%$ after 10 iterations, and it reaches to $65 \%$ at 50 iterations.

Changing mindsets. We also study the effect of changing mindsets between consecutive iterations. We consider three different values for the $\theta$ parameter: $0.2,0.5$, and 0.8 . The larger values of $\theta$ increase the probability that mindsets stay unchanged between consecutive iterations. Bottom row in Figure ?? shows the results. We observe that both extremely low and high values of $\theta$ lead lower values of HR compared to the other case. The effect is even amplified in more iterations. We conclude that the optimal strategy for changing mindsets is a mixed strategy, i.e., the mindsets do not necessarily change at each iteration, but there is an equal chance that the previous mindset is employed again.
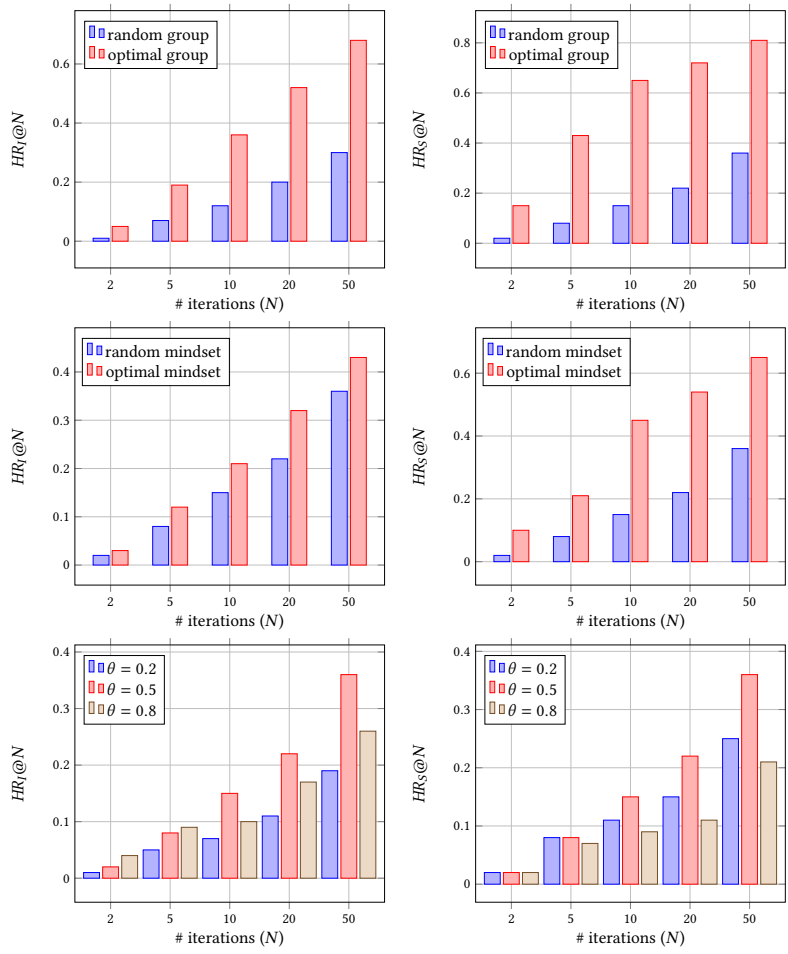

Figure 3: Execution time of LikeMind.

\subsection{User Study}

In this section, we discuss human-oriented aspects of LikeMind. We perform an extensive between-subject user study in Amazon Mechanical Turk ${ }^{9}$ (AMT) to measure the effectiveness of employing look-alike groups and mindsets in LikeMind. Appendix D provides details of this study.

5.2.1 Comparative study. In the first part of our study we compare LikeMind with the following baselines: Diversity, Popularity, and Google Map Explore, i.e., the most popular map navigation application ${ }^{10}$. Each baseline returns $k \times k^{\prime}$ POIs. The participant observes the results of LikeMind and each baseline side-by-side, and should decide which set of POI recommendations is more useful. To remove bias, we don't reveal the identity of the method, and shuffle the order of their appearance for each participant. We found out that $98 \%, 87 \%$, and $59 \%$ of the participants prefer LikeMind over Diversity, Popularity and Google Map Explore, respectively. While the identity of the methods were concealed, the participants have most probably used Google Map Explore before, but not LikeMind. This a-priori familiarity could be an influencing factor in this study, which led many participants to vote on their "comfort zone" technological method.

5.2.2 Independent study. In the next part of the user study, we delve into more details of evaluating look-alike groups and mindsets in

\footnotetext{
${ }^{9}$ https://www.mturk.com

${ }^{10} \mathrm{https} / / /$ themanifest.com/mobile-apps/popularity-google-maps-trends-navigationapps-2018
} 

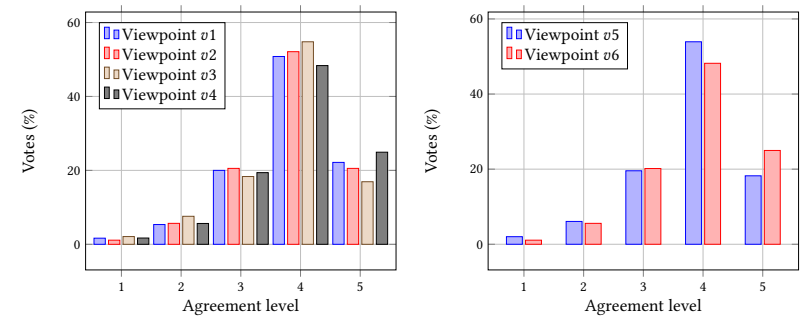

Figure 4: LikeMind user study.

LikeMind. For each participant in our study, we describe a context (time and location, e.g., "an evening in Paris") and a mindset. Then we show the results of LikeMind ( $k$ groups and their $k^{\prime}$ POIs) and ask the participant's opinion about two different aspects of our system: group-based and interaction-based. The former relates to the usefulness of look-alike groups in POI recommendation. The latter is also about the usefulness of a multi-shot recommender where participants can enrich their intent in several iterations. For both aspects, we pose a set of viewpoints, where the participant expresses his/her agreement with each viewpoint in a Likert scale with the following scores: 1 (totally disagreed), 2 (disagreed), 3 (neutral), 4 (agreed), and 5 (totally agreed).

Usefulness of look-alike groups. Figure 4-left reports the groupbased aspects. The first two viewpoints evaluate the overall usefulness of look-alike groups in POI recommendation: $(v 1)$ the groupbased results are relevant to the selected mindset, (v2) the groups help the participant understand why he/she receives the POIs as recommendation results. For both viewpoints, we observe that the supremacy of the agreement vote is statistically significant. This validates our hypotheses on the relevance of group-based recommendations to the mindset (i.e., viewpoint $v 1$ ) and the understandability of recommended POIs using groups (i.e., viewpoint $v 2$ ). We conducted one-way repeated ANOVA for the both results, and obtained the $F$ statistics of 311.97 and 731.30 with the significance level of 0.05 , for $v 1$ and $v 2$ respectively.

Usefulness of group descriptions. The viewpoints $v 3$ and $v 4$ evaluate the usefulness of group descriptions in the recommendation process: ( $v 3)$ reading group descriptions helps the participant in deciding about recommended POIs, $(v 4)$ the group descriptions are relevant to the recommended POIs. In $v 3$, we observe that the majority of votes belongs to the agreement side, i.e., $50.81 \%$ agreed, and $22.16 \%$ strongly agreed. Regarding $v 4$, while $52.11 \%$ of participants voted "agreed", there is a tie between "neutral" and "totally agreed" at $20.54 \%$. However, the agreement vote is statistically significant in both results, with $F$ statistics of 700.17 and 499.35 , for $v 3$ and $v 4$ respectively (using one-way repeated ANOVA with the significance level of 0.05). We also asked our participants which part of the group description is more helpful in enabling the decision making about recommended POIs. We found that $55.11 \%$ of our participants vote "POI categories" as the most helpful content in group descriptions, followed by $34.14 \%$ for "visitor common demographics", and $10.74 \%$ for "time-based information".
Usefulness of interactions. Figure 4-right reports the interactionbased aspects. Each participant is asked to navigate from one iteration to another, either by choosing a new mindset or preserving the current one. We consider two viewpoints for the interactions: (v5) The step-by-step interaction is useful for the user to follow his/her interests, (v6) the iterative process helps the user to reach a decision on which place he/she would like to visit. We observe $72.15 \%$ and $48.43 \%$ of agreement votes, and less than $10 \%$ of disagreement votes, for $v 5$ and $v 6$ respectively. This shows the indispensable need of an iterative process for POI recommendation.

Usefulness of mindsets. Beyond agreement scores on viewpoints, we asked our participants' opinion about resulting groups and POIs per mindset. They could describe their perspective in form of a free text. An extract of these comments is listed in Table 5. For instance, they mentioned that the mindset $m_{1}$ ("I'm new here") is useful for tourism, $m_{2}$ ("surprise me") enables access to out-of-thebox places, and $m_{7}$ ("hidden gems") helps to spot places which are not popular but intriguing. While in general mindsets are found to be helpful, the participants mentioned the lack of POI details as an inconvenience. One of our future directions is to enrich POI attributes by integrating exogenous data sources which describe different aspects of POIs.

\section{RELATED WORK}

To the best of our knowledge, LikeMind is the first customized, contextualized and explainable POI recommendation system to tackle cold start to its full extent. However, our approach does relate to some other approaches in the literature, which we review in this section.

Interactive recommendation. Interactive recommendation is a crucial need for serving cold users and describing complex information needs [31]. Each iteration of an interactive recommendation system constitutes a conversation, where the system provides some information to the user, and the user responds in the form of a feedback. Interactive recommendation is often modeled either as a two-phase approach (e.g., interview-based [23], onboarding [32], active learning [33]) or as an exploratory data analysis (EDA) approach [34]. Most interactive models require users to either go through a tedious knowledge acquisition process, or deal with a complex feedback capturing mechanism. To the best of our knowledge, while LikeMind counts as an EDA approach, it is the first interactive POI recommendation method which provides a lightweight feedback capturing mechanism to guide users through the iterative conversations.

Explainable recommendation. The task of explainable recommendation is to inform users why they receive certain POIs as recommendation results. Most explainability approaches focus on explaining latent representations in ML models in a non-intrusive way $[35,36]$, where the internals of the systems remain unexplained. For an enhanced explainability, it is required to either feed some ontological a-priori knowledge to the process, or rebuild an explainable structure from the ground up. LikeMind is a transparency-bydesign method where recommendations are naturally explainable using look-alike groups. While it is shown in [37] that feature explanation is among the most challenging aspects of explainable 


\begin{tabular}{|c|c|}
\hline Mindset & Comments \\
\hline$m_{1}:$ I'm new here & $\begin{array}{l}\text { "I find this helpful, because most people have similar interests on tourism." "The group } \\
\text { description allows you to follow a group that conforms to your tastes." I love to travel, so } \\
\text { these recommendations would be great, considering that I've never been to that location before." } \\
\text { "When visiting a new country, I like to take recommendations from others on where to go } \\
\text { and what to see." "I appreciate the way the system categorized the locations because it allows } \\
\text { me to understand what type of curiosity it might fulfill." }\end{array}$ \\
\hline$m_{2}:$ surprise me & $\begin{array}{l}\text { "Since I was interested in visiting places unlike what I had seen before, I believe it makes } \\
\text { the most sense to base my selections off of the proposed groups." "Surprise me' would be } \\
\text { helpful to just pick a place and go." "The proposed groups seemed to offer out-of-the-box } \\
\text { places to visit, which went with my interest." }\end{array}$ \\
\hline$m_{3}$ : let's workout & $\begin{array}{l}\text { "I would want to go where other people found the most invigorating exercise that burned the } \\
\text { most calories." "These are diverse adventures which fit my energetic personality." } \\
\text { "I appreciate that the system is showing me where I can exercise and do physical activities } \\
\text { like biking." "I like physical activities but also enjoy eating a healthy meal or going to a park } \\
\text { to walk and get some exercise. So this was helpful to me that the system showed me places } \\
\text { where I can do that based on group visits/recommendations." }\end{array}$ \\
\hline$m_{4}:$ me time & $\begin{array}{l}\text { "The groupings would help me avoid overly busy places if I want to relax." "I needed some } \\
\text { 'me time' and felt that the Village Saint Paul was the best for me, with cobblestone streets, } \\
\text { a nice museum and a quaint coffee shop." "A good coffeehouse or café could be helpful, } \\
\text { especially at a specific time of day, or if it is a place favored by locals." }\end{array}$ \\
\hline$m_{5}: I^{\prime} \mathbf{r}$ & $\begin{array}{l}\text { "It is helpful, as you can see at a glance what restaurants and coffee shops are around you." } \\
\text { "I like that the places were in close proximity for meal choices." "With regards to food } \\
\text { preferences, the common categories are good descriptors, because they show a little bit } \\
\text { about what kind of food experience (mainstream or local) that a person would want." }\end{array}$ \\
\hline$m_{6}:$ let's learn & $\begin{array}{l}\text { "It gave me a chance to catch up on history." "It allows you to focus on more museum-oriented } \\
\text { travelers." "Since I was looking for learning opportunities, I believe it was the most useful to look } \\
\text { at the common categories of places that group members visit. "These places are exactly what I } \\
\text { would visit Paris for." "I was able to pick out places where I could learn more about the history } \\
\text { of Paris." }\end{array}$ \\
\hline$m_{7}$ : hidden gems & $\begin{array}{l}\text { "I found this helpful because the places are not very popular, but very intriguing and interesting." } \\
\text { "I love the recommendations which are not common place" "I believe that the demographics were } \\
\text { the most useful in this scenario. To me, the individuals with many friends would be able to 'get } \\
\text { the scoop' on hidden gems in the area." "This helped to acknowledge smaller but good places } \\
\text { to visit that may have otherwise been missed." "If I was looking for hidden gems, I would prefer to } \\
\text { go somewhere not frequented by other people at all." }\end{array}$ \\
\hline
\end{tabular}

Table 5: Participants' opinions about resulting groups and POIs for each mindset

recommendation, look-alike groups easily manifest the set of features that made a POI set recommendable.

Look-alike groups. To tackle the challenge of cold start, many approaches are proposed to enrich a sparse check-in matrix with social aspects. The assumption is that users may be more interested to visit places that their friends had visited in the past [12]. In [38], a friendship-based CF is proposed. In [39], the Matrix Factorization model is extended with social regularization. Moreover, friendship links are exploited in [40] to build friend groups using Community Detection techniques. In LikeMind, we extend the domain of cold start to social aspects, i.e., no friendship link is available for users. LikeMind builds explainable look-alike groups without relying on any social aspects or check-ins of the user.

Mindsets. Mindsets capture user needs. Category-based search interfaces $[41,42]$ capture explicit needs of users in the form of categories (e.g., selecting POIs of the category "historical landmarks").
Categories enable more personalization for users, resulting in less anxiety and more trust over the system. However, realistic scenarios often contain ambiguous needs and intents, where users seek to disambiguate in an iterative process. The only possible iteration in traditional search paradigms is to restart a search with another category. Exploratory travel interfaces [43] have been found to enhance user experience in POI exploration with serendipity measures. In LikeMind, we employ "mindsets", which is an intuitive way of capturing user's implicit intents. Mindsets reflect interests of users, and align to users' preferences during iterations.

\section{CONCLUSION}

We present LikeMind, an interactive and explainable POI recommendation system based on look-alike groups, which tackles the common challenges of cold start, customizability, contextuality, and explainability. We introduce the notion of "mindsets" which extends user context, and captures actual situation and intents of 
the user. In an extensive set of experiments, we show that LikeMind achieves a Hit Ratio higher than $50 \%$ only after 10 iterations. In an extensive user study, we showed the effectiveness of look-alike groups and mindsets for POI recommendation.

We have several directions to pursue as future work. We plan to integrate planning and mobility aspects in LikeMind. While our focus in the current paper was on the actual context of the user, the functionality of the system can be enriched with some user-defined constraints on POI preferences and mobility patterns.

\section{ACKNOWLEDGEMENT}

We thank Antonietta Grasso, Adrien Bruyat, Cécile Boulard, and Denys Proux, for their valuable comments and feedback.

\section{REFERENCES}

[1] Hangil Chung, Daniel Freund, and David B Shmoys. Bike angels: An analysis of citi bike's incentive program. In SIGCAS. ACM, 2018.

[2] Kaiyu Feng, Gao Cong, Sourav S Bhowmick, Wen-Chih Peng, and Chunyan Miao. Towards best region search for data exploration. In SIGMOD, 2016.

[3] Zheng Xu, Yunhuai Liu, Neil Yen, Lin Mei, Xiangfeng Luo, Xiao Wei, and Chuanping $\mathrm{Hu}$. Crowdsourcing based description of urban emergency events using social media big data. TCC, 2016.

[4] Cicero A. L. Pahin, Behrooz Omidvar-Tehrani, Sihem Amer-Yahia, Valerie Siroux, Jean-Louis Pepin, Jean-Christian Botel, and Comba Joao. COVIZ: A system for visual formation and exploration of patient cohorts. In VLDB, 2019.

[5] Jie Bao, Yu Zheng, David Wilkie, and Mohamed Mokbel. Recommendations in location-based social networks: a survey. GeoInformatica, 2015.

[6] Fan Du, Catherine Plaisant, Neil Spring, and Ben Shneiderman. Finding similar people to guide life choices: Challenge, design, and evaluation. In CHI, 2017.

[7] Jeni Paay, Jesper Kjeldskov, Mikael B Skov, Per M Nielsen, and Jon Pearce. Discovering activities in your city using transitory search. In Mobile-CHI, 2016.

[8] Sarabjot Singh Anand and Bamshad Mobasher. From web to social web: Discovering and deploying user and content profiles. chapter Contextual Recommendation. Springer-Verlag, 2007.

[9] Justin J Levandoski, Mohamed Sarwat, Ahmed Eldawy, and Mohamed F Mokbel. Lars: A location-aware recommender system. In ICDE, 2012.

[10] Bin Liu, Yanjie Fu, Zijun Yao, and Hui Xiong. Learning geographical preferences for point-of-interest recommendation. In SIGKDD, 2013.

[11] Hongzhi Yin, Bin Cui, Yizhou Sun, Zhiting Hu, and Ling Chen. Lcars: A spatial item recommender system. TOIS, 2014.

[12] Hao Wang, Manolis Terrovitis, and Nikos Mamoulis. Location recommendation in location-based social networks using user check-in data. In SIGSPATIAL, 2013.

[13] Huayu Li, Yong Ge, Richang Hong, and Hengshu Zhu. Point-of-interest recommendations: Learning potential check-ins from friends. In SIGKDD, 2016.

[14] Hao Wang, Manolis Terrovitis, and Nikos Mamoulis. Location recommendation in location-based social networks using user check-in data. In SIGSPATIAL, 2013.

[15] Shagun Jhaver, Yoni Karpfen, and Judd Antin. Algorithmic anxiety and coping strategies of airbnb hosts. In CHI, page 421. ACM, 2018.

[16] Liqiang Geng and Howard J Hamilton. Interestingness measures for data mining: A survey. ACM Computing Surveys (CSUR), 38(3):9, 2006.

[17] Arnab Nandi and HV Jagadish. Guided interaction: Rethinking the query-result paradigm. VLDB, 4(12):1466-1469, 2011.

[18] Behrooz Omidvar-Tehrani and Sihem Amer-Yahia. User group analytics: Survey and research opportunities. TKDE, 2019.

[19] Sruthi Viswanathan, Behrooz Omidvar-Tehrani, Adrien Bruyat, Frédéric Roulland, and Maria Antonietta Grasso. Hybrid wizard of oz: Concept testing a recommender system. In CHI 2020, pages 1-7, 2020.

[20] Waldo R Tobler. A computer movie simulating urban growth in the detroit region. Economic geography, 46(sup1):234-240, 1970.

[21] Ramesh Baral, S. S. Iyengar, Tao Li, and XiaoLong Zhu. Hicaps: Hierarchical contextual poi sequence recommender. In SIGSPATIAL, 2018.

[22] Ting Shen, Haiquan Chen, and Wei-Shinn Ku. Time-aware location sequence recommendation for cold-start mobile users. In SIGSPATIAL, 2018.

[23] Sihem Amer-Yahia, Ria Mae Borromeo, Shady Elbassuoni, Behrooz OmidvarTehrani, and Sruthi Viswanathan. Interactive generation and customization of travel packages for individuals and groups. In IUI '20, pages 69-70. ACM, 2020.

[24] Radi Muhammad Reza, Mohammed Eunus Ali, and Muhammad Aamir Cheema. The optimal route and stops for a group of users in a road network. In SIGSPATIAL, pages $1-10,2017$.

[25] Lionov Wiratma, Marc van Kreveld, Maarten Löffler, and Frank Staals. An experimental evaluation of grouping definitions for moving entities. In SIGSPATIAL, page 89âĂŞ98, 2019.
[26] Udo Feuerhake and Monika Sester. Mining group movement patterns. In SIGSPATIAL, 2013.

[27] Ilhem Feddaoui, Faîçal Felhi, and Jalel Akaichi. Extract: New extraction algorithm of association rules from frequent itemsets. In ASONAM, pages 752-756. IEEE, 2016.

[28] Jean-Daniel Fekete, Danyel Fisher, Arnab Nandi, and Michael Sedlmair. Progressive data analysis and visualization (dagstuhl seminar 18411). Dagstuhl Reports, 8 (10):1-40, 2018. doi: 10.4230/DagRep.8.10.1. URL https://doi.org/10.4230/DagRep. 8.10 .1

[29] Behrooz Omidvar-Tehrani, Sihem Amer-Yahia, and Ria Mae Borromeo. User group analytics: hypothesis generation and exploratory analysis of user data. VLDB 7., 28(2):243-266, 2019.

[30] Yong Liu, Wei Wei, Aixin Sun, and Chunyan Miao. Exploiting geographical neighborhood characteristics for location recommendation. In CIKM, pages 739-748. ACM, 2014.

[31] Filip Radlinski and Nick Craswell. A theoretical framework for conversational search. In CHIIR, pages 117-126, 2017.

[32] Konstantina Christakopoulou, Alex Beutel, Rui Li, Sagar Jain, and Ed H Chi. Q\&R: A two-stage approach toward interactive recommendation. In $K D D$, pages 139-148, 2018.

[33] Kyriaki Dimitriadou, Olga Papaemmanouil, and Yanlei Diao. AIDE: an active learning-based approach for interactive data exploration. IEEE Trans. Knowl. Data Eng., 28(11):2842-2856, 2016.

[34] Behrooz Omidvar-Tehrani, Sihem Amer-Yahia, and Alexandre Termier. Interactive user group analysis. In CIKM, pages 403-412, 2015.

[35] Yongfeng Zhang, Guokun Lai, Min Zhang, Yi Zhang, Yiqun Liu, and Shaoping Ma. Explicit factor models for explainable recommendation based on phrase-level sentiment analysis. In SIGIR, pages 83-92, 2014.

[36] Nan Wang, Hongning Wang, Yiling Jia, and Yue Yin. Explainable recommendation via multi-task learning in opinionated text data. In SIGIR, pages 165-174, 2018.

[37] Iordanis Xanthopoulos, Ioannis Tsamardinos, Vassilis Christophides, Eric Simon, and Alejandro Salinger. Putting the human back in the automl loop. In EDBT/ICDT Workshops, 2020

[38] Mao Ye, Peifeng Yin, Wang-Chien Lee, and Dik-Lun Lee. Exploiting geographical influence for collaborative point-of-interest recommendation. In SIGIR, pages 325-334. ACM, 2011

[39] Mohsen Jamali and Martin Ester. A matrix factorization technique with trust propagation for recommendation in social networks. In Proceedings of the fourth ACM conference on Recommender systems, pages 135-142. ACM, 2010.

[40] Deepika Lalwani, Durvasula VLN Somayajulu, and P Radha Krishna. A community driven social recommendation system. In Big Data, 2015.

[41] Marti Hearst. Search user interfaces. Cambridge university press, 2009.

[42] Yan Lyu, Chi-Yin Chow, Ran Wang, and Victor C. S. Lee. Using multi-criteria decision making for personalized point-of-interest recommendations. In SIGSPATIAL, 2014.

[43] Sruthi Viswanathan, Cecile Boulard, and Antonietta Maria Grasso. Ageing clouds: Novel yet natural support for urban exploration. In DIS '19 Companion, pages 313-317, 2019.

\section{APPENDIX}

\section{A NP-COMPLETENESS PROOF}

Theorem 1. The POI recommendation problem defined in Section 3.3 is NP-Complete.

Proof. (sketch) It is shown in [29] that the problem of picking $k$ groups by maximizing an optimization objective is NP-Complete by a reduction from Maximum Edge Subgraph (MES) problem. In the problem of POI recommendation using look-alike groups, we have two additional elements: (i) relevance and distance constraints, and (ii) maximization of more than one objective (as mindset functions combine several utility functions as objectives). This means that the problem in [29] is a special case of ours. Hence our problem is obviously harder.

\section{B TRANSACTION EMBEDDINGS}

Transaction embeddings are encoded as integers. Hence all POIs and demographic values should be encoded to single integers. While POIs are often already associated to a unique identifier which can be directly used in the embeddings, demographic attributes may have 
different values in discrete or continuous domains. For instance, the demographic attribute "number of check-ins" may contain different values in a wide range. We employ an equal-frequency discretization approach to obtain 4 categories for each demographic attribute. Table 6 illustrates discretization values for demographic attributes in Gowalla. The discretized attributes result in 28 items (7 attributes and 4 categories for each) to be concatenated to the embeddings. For instance, a visitor embedding may contain items "many places" and "few photos", which means that the visitor went to many places, but did not take many pictures.

\begin{tabular}{|l|l|l|l|l|}
\hline & very few & few & some & many \\
\hline \hline items & $\leq 2$ & $(2,3]$ & $(3,5]$ & $\geq 5$ \\
\hline photos & $\leq 1$ & $(1,2]$ & $(2,5]$ & $\geq 5$ \\
\hline friends & $\leq 1$ & $(1,3]$ & $(3,5]$ & $\geq 5$ \\
\hline check-ins & $\leq 3$ & $(3,12]$ & $(12,34]$ & $\geq 34$ \\
\hline places & $\leq 3$ & $(3,9]$ & $(9,23]$ & $\geq 23$ \\
\hline
\end{tabular}

Table 6: Equal-frequency discretization of demographic attributes in Gowalla dataset.

Beyond demographics and visited POIs, we enrich the embeddings by POI categories and check-in time. For a POI $p$ in an embedding of a visitor $u$, we concatenate p.att to $\operatorname{trans}(u)$. For instance, if Louvre $\in \operatorname{trans}(u)$, we also consider $\langle$ cat, museum $\rangle$ as an additional item in $\operatorname{trans}(u)$. This enables the system to generate a group of visitors who check in museums in general, but not necessarily Louvre. For instance in Figure 1, the visitors of the green group have all checked in a "historical landmark", but it could be different landmarks that they had checked in. Also for a pair of POI and visit time $\langle p, t\rangle \in u$.checkins, we discretize $t$ to hourly categories ("morning", "afternoon", "evening", and "night") and weekly categories ("weekday" and "weekend") and concatenate $\operatorname{trans}(u)$ with those hourly and weekly categories alongside $p$ 's category. For instance in Figure 2, members of the green group have all checked in a restaurant (any POI with the category "restaurant") in the evening.

\section{PERFORMANCE STUDY}

In addition to the simulation study (Section 5.1) and the user study (Section 5.2), we evaluate the efficiency of LikeMind by measuring the average execution time at each iteration. We conjecture that the radius $r$ and the number of groups $k$ are the two most influencing input parameters on the performance of LikeMind. Hence we report the execution time by varying $r$ between $50 \mathrm{~m}$ and $1 \mathrm{~km}$, and $k$ between 5 and 70 . Note that we don't analyze the $k^{\prime}$ parameter, as it is a dependent variable to $k$. All the performance experiments are conducted on an $2.2 \mathrm{GHz}$ Intel Core i7 with 32GB of DDR4 memory on OS X 10.14.6 operating system.

To dissect LikeMind's performance, we report the execution time for each of the following stages of Algorithm 1: stage 1: find nearby POIs (nearby_POIs() function in line 1 of the algorithm), stage 2: find check-ins of the nearby POIs (checkins_of () function in line 2), stage 3: build the transaction matrix and generate look-alike groups (mine_groups() function in line 3), and stage 4: find $k$ optimal groups and their $k^{\prime}$ associated POIs regarding a given mindset (maximize() function in line 4). We report the average execution time over 100 iterations. Each iteration is simulated as described in Section 5.1. However note that we don't analyze sessions in the performance
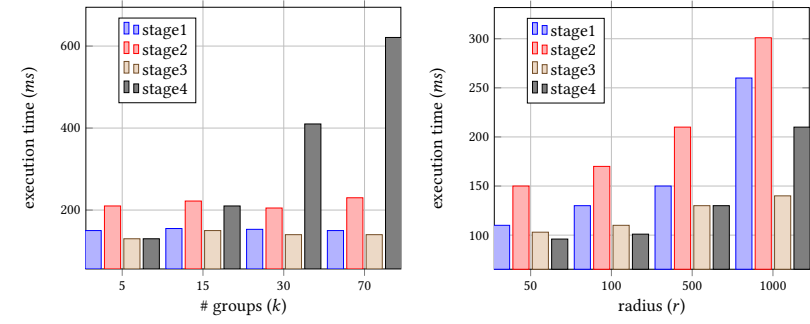

Figure 5: Execution time of LikeMind.

study, and we consider that each iteration is independent from others. By default, we consider $k=k^{\prime}=5$, and $r=0.5 \mathrm{~km}$.

Figure 5 depicts the results of our performance study. The left and right charts report execution time by varying $k$ and $r$, respectively. We observe that increasing $k$ only influences stage 4 (i.e., mindset maximization), and the execution time of all other stages stays constant. The average execution time for the three first stages are $152 \mathrm{~ms}, 216.25 \mathrm{~ms}$, and $140 \mathrm{~ms}$, respectively. The time for executing the fourth stage grows from $130 \mathrm{~ms}$ at $k=5$ to $621.75 \mathrm{~ms}$ at $k=70$. Note that while the time limit $t l$ is always fixed to $100 \mathrm{~ms}$ (see Section 4), stage 4 requires extra time to obtain $k^{\prime}$ POIs for each group, generate output, and save it on disk. While $k$ is oblivious to the three first stages of LikeMind, increasing $r$ influences the execution time of all the stages. The reason is that a larger radius implies that more POIs and consequently more check-ins should be retrieved from the database. Abundance of check-ins makes the group candidate space larger, hence the group generation stage requires more time. The overall execution time of LikeMind grows from $459 \mathrm{~ms}$ at $r=50 \mathrm{~m}$ to $911 \mathrm{~ms}$ at $r=1 \mathrm{~km}$. We consider $r=500 \mathrm{~m}$ as the default value whose collective execution time is on average $620 m s$.

\section{USER STUDY}

We recruited 753 participants in AMT and forwarded them to a Survey Monkey questionnaire ${ }^{11}$ to answer different questions about the functionality of our proposed system. $51 \%$ of the participants were female. Also the majority of them was in the age range of 25-34 (44.09\%) followed by the age ranges $34-44$ and $18-24$ by $20.22 \%$ and $18.04 \%$, respectively. Moreover, $54 \%$ of them were from the US, $22 \%$ from India, and $24 \%$ from the rest of the world. Each participant received $\$ 0.07$ as the incentive to complete the study. To make the results of our study easily interpretable and comparable, we consider a controlled environment where the participants always select one of the mindsets in Table 2 and won't propose a new mindset (mindset creation is discussed in Section 4.4). The user study for mindset creation is a part of our future work.

Before the user study. Before we show LikeMind results to the participants, we perform a pre-test to identify motivations behind using a POI recommender. The participants should select at least one among 5 pre-defined options depicted in Table 7 . We observe that the dominant motivations are "knowing more about places" and "spending time with others". The exploratory nature of LikeMind

\footnotetext{
${ }^{11}$ https://www.surveymonkey.com
} 

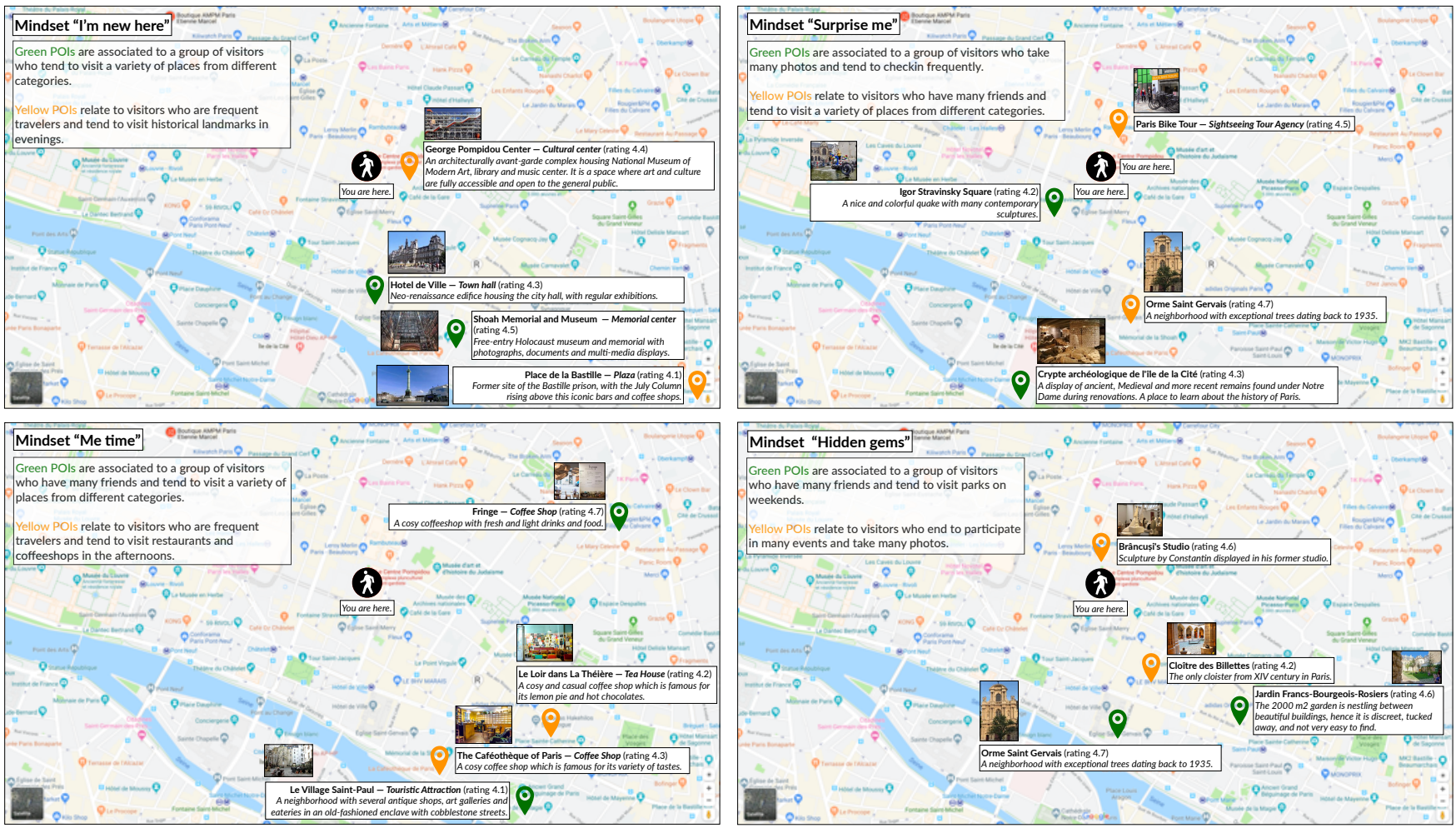

Figure 6: An example of POIs provided in the user study.

helps users to know better their POI options in several iterations. Also look-alike groups help them to receive an explanation for the recommendations, and enable them to decide better about where they can go with their company. The third dominant option is "wellbeing", which stresses on the specificity of users' intents. Mindsets in LikeMind are designed to capture such intents. For instance, by selecting the mindsets $m_{3}$ (let's workout) and $m_{4}$ (me-time), the user will receive recommendations which optimize his/her physical and mental well-being, respectively.

\begin{tabular}{|l|l|}
\hline Option & Vote (\%) \\
\hline \hline know more about places & 58.38 \\
\hline spend time with friends and family & 56.48 \\
\hline well-being (mental and physical) & 37.92 \\
\hline post stories and photos on Instagram & 20.71 \\
\hline boredom & 16.96 \\
\hline
\end{tabular}

Table 7: Motivations for using a POI recommender
During the user study. The pre-test helped us design AMT tasks for our user study based on users' needs. Given a mindset $m$, the participants receive $k$ groups and $k^{\prime}$ POIs optimized for $m$. In comparative studies, $k \times k^{\prime}$ POIs of a baseline will be also illustrated side-by-side. Figure 6 shows examples of POIs that the participants received during our user study, for the mindsets "I'm new here", "surprise me", "me time", and "hidden gems" $\left(k=k^{\prime}=2\right)$. All POIs are at most one kilometer far (i.e., $r=1 \mathrm{~km}$ ) from George Pompidou Center in Paris (i.e., the user's location). As AMT participants may not know Paris, each POI is annotated with a photo, rating, and a concise description, using Google Places $\mathrm{API}^{12}$.

\footnotetext{
${ }^{12}$ cloud.google.com/maps-platform/places
} 